\title{
A stochastic model of stroma: interweaving variability and compressed fibril exclusion
}

Marcello Vasta ${ }^{1}$, Alessio Gizzi², Anna Pandolfi ${ }^{3}$

${ }^{1}$ Department of Engineering and Geology, University of Chieti-Pescara, Chieti, Italy; ${ }^{2}$ Department of Engineering, University Campus Bio-Medico of Rome, Rome, Italy; ${ }^{3}$ Departament of Civil and Environmental Engineering, Politecnico di Milano (Polytechnic University of Milan), Milan, Italy

\section{Abstract}

Hyperelastic constitutive models of the human stroma accounting for the stochastic architecture of the collagen fibrils and particularly suitable for computational applications are discussed. The material is conceived as a composite where a soft ground matrix is embedded with collagen fibrils characterized by non-homogeneous spatial distributions typical of reinforcing stromal lamellae. A multivariate probability density function of the spatial distribution of the fibril orientation is used in the formulation of the lamellar branching observed on the anterior third of the stroma, selectively excluding the contribution of compressed fibrils. The physical reliability and the computational robustness of the model are enhanced by the adoption of a second order statistics approximation of the average structure tensors typically employed in fiber reinforced models.

Keywords: compressive fibril exclusion, hyperelasticity, lamellar branching, second order structure tensors

\section{Introduction}

The cornea is the external lens of the eye and carries out concerted protective, structural, and refractive functions. Since the cornea provides one-third of the total

Correspondence: M. Vasta, Dipartimento INGEO, Università di Chieti-Pescara, Viale Pindaro 42, Pescara, Italy.

E-mail:mvasta@unich.it 
refractive power of the eye and due to its accessibility, it stands as the privileged site for surgical interventions to correct conditions such as myopia, presbyopia, and astigmatism. Corneal refractive surgery demands accurate diagnostic and surgical plans, but the outcomes are not always optimal. ${ }^{1}$ Advanced numerical models of the cornea have been developed in recent years with the aim of supporting refractive surgery, ${ }^{2,3}$ although they are still far to be an active part of the current clinical practice since they fail to be patient-specific models. Indeed, patient-specific models derive only from the combination of advanced diagnostic imaging, correct interpretation of optical and mechanical experimental observations, and efficient computational models. For one, the interpretation of the most promising in-vivo dynamical test (contactless air-puff tonometer) calls for the description of features of the stromal tissue that are not of relevance under more traditional quasistatic loading. The present study is thus concerned with the definition of accurate material models of the stromal tissue, based on the description of the collagen fibril architecture according to its statistical distribution, characterized by a different degree of interweaving across the thickness ${ }^{4}$ and inactivity of the fibrils under compressive loading. Starting from a second order approximation of the strain energy density of a statistical distribution of collagen fibrils, ${ }^{5}$ a multivariate probability density function (PDF) ${ }^{6}$ is introduced to incorporate recent experimental data. ${ }^{7}$

\section{Hyperelastic fibril distributed models}

\subsection{Second order structure tensor approach}

Stochastic models for fiber reinforced tissues ${ }^{8}$ derive from the seminal work of Lanir, ${ }^{9}$ where the contribution of fiber orientation to the mechanical response of soft tissues is formulated from a theoretical point of view. The computational challenge of describing the microstructural properties in a reliable manner motivated the development of several approximations..$^{10-18}$ The present study departs from the second order approximation of the strain energy density $\psi_{f}^{*}$ of the collagen fiber distribution illustrated in Pandolfi and Vasta. ${ }^{5}$ The approximation is based on the second order Taylor expansion of the fiber strain energy density about the fourth pseudo-invariant ${\overline{T_{4}}}_{4}=\overline{\mathbf{C}}: \mathbf{A}$, where $\mathbf{A}=\mathbf{a} \otimes \mathbf{a}$ is the structure tensor associated to the mean direction a of the fiber distribution, i.e., $\psi$ :

$$
\psi_{f}^{*} \simeq \psi_{f}^{0}+\bar{\Psi}_{f}^{*}\left(1+\mathrm{K}^{\star} \sigma_{I_{4}}^{2}\right)
$$

with

$$
\begin{aligned}
& \Psi_{f}^{0}=-\frac{k_{1}}{2 k_{2}} \quad \Psi_{f}^{*}=\frac{k_{1}}{2 k_{2}} \exp \left[k_{2}\left(I_{4}^{*}-1\right)^{2}\right] \\
& I_{4}^{*}=\left\langle I_{4}\right\rangle=\boldsymbol{H}: \overline{\mathbf{C}}, \sigma_{I_{4}}^{2}=\left\langle\left(I_{4}-I_{4}^{*}\right)^{2}\right\rangle=\mathbb{H}: \overline{\mathbf{C}}
\end{aligned}
$$


and $\boldsymbol{H}$ and $\mathbb{H}$ define the second and forth order averaged structure tensors, respectively. The model provides a closed form expression of the second Piola-Kirchhoff stress tensor:

$$
\overline{\mathrm{S}}_{\mathrm{f}}^{\star} \simeq \alpha\left(I_{4}^{\star}, \sigma_{l_{4}}^{2}\right) \boldsymbol{H}+\beta\left(I_{4}^{\star}, \sigma_{l_{4}}^{2}\right) \mathbb{H}: \overline{\mathbf{C}}
$$

which is effective and robust in computational applications. ${ }^{14,15,18}$

\subsection{Computational modeling of interweaving variability}

The collagen fibril architecture of the stroma is characterized by a regular variation across the thickness, with a marked interweaving in the anterior third and a predominant surface tangent (planar) orientation distribution in the posterior third. ${ }^{4,7}$ The organization of the fibrils can be modelled through a multivariate von Mises distribution, ruling both the in-plane and transverse orientation of the fibrils. Using the spherical angles $[\Theta, \Phi] \in[0, \pi],[0, \pi]$ the generic orientation $\boldsymbol{a}$ is:

$$
\boldsymbol{a}=\sin \Theta \cos \phi \mathrm{e}_{1}+\sin \Theta \sin \phi \mathrm{e}_{2}+\cos \Theta \mathrm{e}_{3}
$$

The corresponding PDF, $\rho(\alpha)$, decomposes in out-of-plane term $\rho_{\theta}(\theta)$ and in-plane term $\rho \phi(\phi)$ as $\rho(\mathbf{a})=\rho \theta(\theta) \rho \phi(\phi)$ :

$$
\begin{aligned}
& \rho \theta(\theta)=N_{\theta} \exp \left[b_{\theta} \cos 2 \theta\right] \\
& \rho \phi(\phi)=N_{\phi} \exp \left[b_{\phi} \cos (\phi-\pi / 2)\right]
\end{aligned}
$$

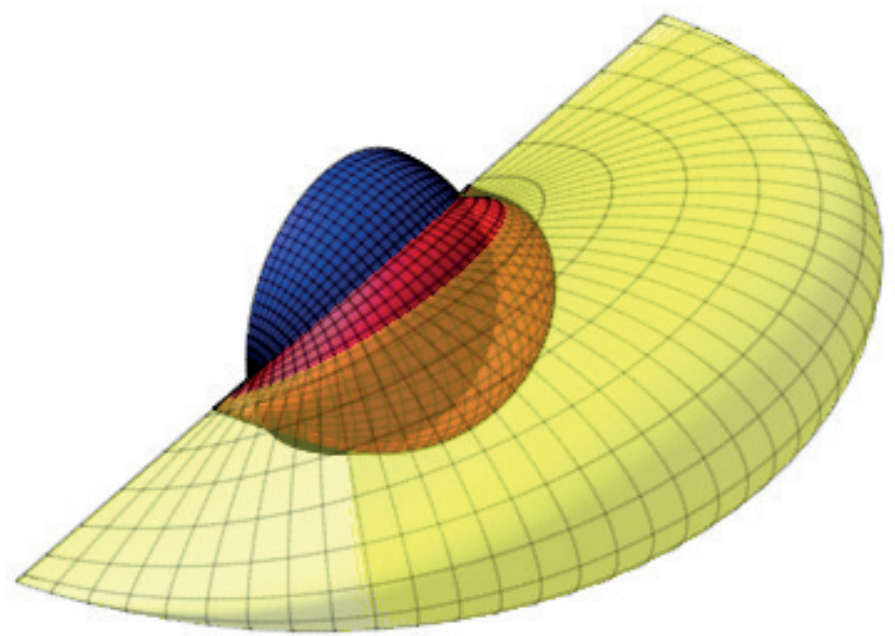

Fig. 1. Representative example of the multivariate von Mises PDF for three different combinations of the material parameters, $b_{\theta}, b_{\phi}$. 

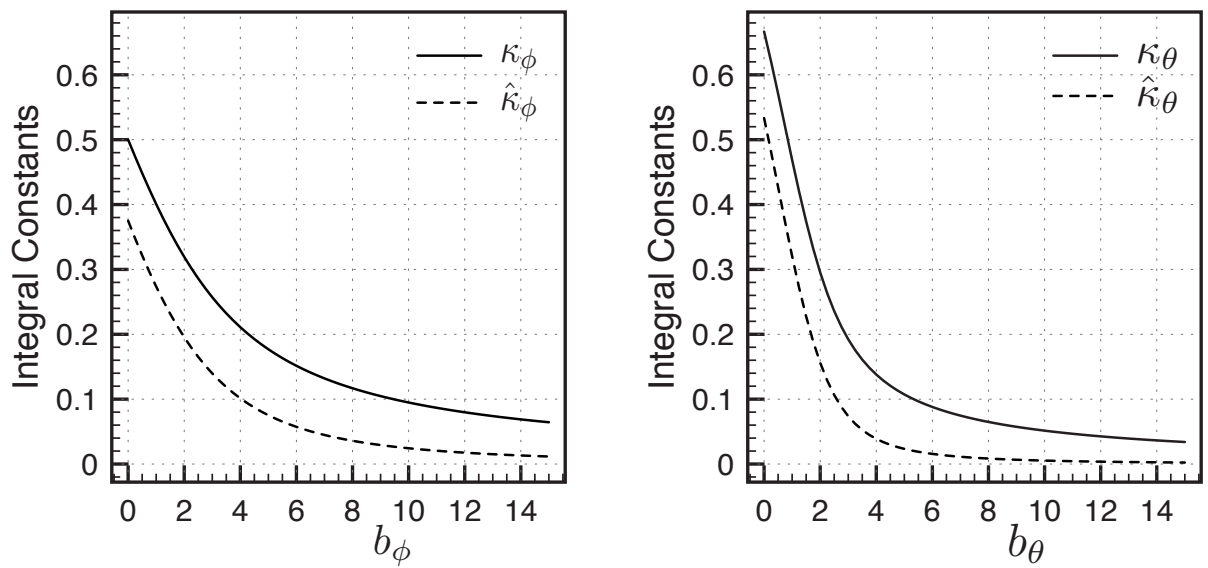

Fig. 2. Representative example of the integral coefficients derived from the multivariate PDF varying with the parameters $\mathrm{b}_{\theta}$ and $\mathrm{b}_{\phi}$. (Left) $\mathrm{k}_{\theta}, \widehat{\mathrm{k}}_{\theta}$ versus $b_{\theta}$. (Right) $\mathrm{k}_{\phi}, \widehat{\mathrm{k}}_{\phi}$ versus $b_{\phi}$.

The decomposition preserves the simple structure tensor form of the approximated strain energy density. A representative example of multiple combinations is provided in Figure 1.

The formulation allows:

1. to characterize complex spatial PDF by means of only two material parameters, $\mathrm{b}_{\theta}, \mathrm{b}_{\phi}$; and

2. to recover a diagonal form for the averaged structure tensor.

The remarkable computational implication of the approach is that the averaged structure tensors $\boldsymbol{H}$ and $\mathbb{H}$ depend exclusively on four integral coefficients:

$$
\begin{array}{ll}
\mathrm{\kappa}_{\theta}=\frac{1}{N_{\theta}} \int_{0}^{\pi} \rho_{\theta} \sin ^{3} \theta d \theta & \widehat{\mathrm{K}}_{\theta}=\frac{1}{\mathrm{~N}_{\theta}} \int_{0}^{\pi} \rho_{\theta} \sin ^{5} \theta d \theta \\
\mathrm{\kappa}_{\phi}=\frac{1}{N_{\phi}} \int_{0}^{\pi} \rho_{\phi} \sin ^{3} \phi d \phi & \widehat{\mathrm{\kappa}}_{\phi}=\frac{1}{\mathrm{~N}_{\phi}} \int_{0}^{\pi} \rho_{\phi} \sin ^{5} \phi d \phi
\end{array}
$$

A representative example of the novel behavior induced by the multivariate PDF is provided in Figure 2 with respect to different values of the concentration parameters $\mathrm{b}_{\theta}, \mathrm{b}_{\phi}$.

\subsection{Fibril exclusion under compression}

A full statistical approach has a great potential to exclude fibrils in compression. ${ }^{15,19}$ By applying the random variable transformation rule, from the PDF of $\theta, \phi$ it is possible to derive the PDFs of $\bar{I}_{4}, \rho_{I_{4}}\left(I_{4}\right)$ and of $\Psi, \rho_{\psi}(\Psi)$. Moreover, by using a principal reference frame, ${ }^{20}$ it is possible to obtain a closed form expression of $\rho_{l_{4}}\left(I_{4}\right)$ with the 
a priori knowledge of the physical integration range. This notable result leads to the correct statistical exclusion of compressed fibers in the mechanical response of the tissue.

\section{Conclusion}

A material model for fiber reinforced soft materials characterized by stochastic distributions of the fibers is discussed. The approach allows for the description of local smooth variability of the spatial distribution of fibril interweaving, as the one observed across the thickness of the corneal stroma. Moreover, in a principal reference frame description, the knowledge of the portion of compressed fibers is achieved $a$ priori and easily accounted for in numerical calculations. The statistical material model formulation proposed results well suited for multiscale generalization in the context of soft collagenous tissues..$^{21,22}$

\section{Acknowledgements}

We thank the support from the Italian National Group for Mathematical Physics, GNFM-INdAM.

\section{References}

1. Renna A, Pandolfi A, Martinez FC, Aliò JL. Myopic surface ablation in asymmetrical topographies: refractive results and theoretical corneal elastic response. Am J Ophtalmol. 2017;177:34-43.

2. Pandolfi A, Manganiello F. A model for the human cornea: constitutive formulation and numerical analysis. Biomech Mod Mechanobiol. 2006;5:237-246.

3. Simonini I, Pandolfi A. Customized finite element modelling of the human cornea. PLoS One. 2015;10:e0130426.

4. Petsche SJ, Pinsky PM. The role of 3-d collagen organization in stromal elasticity: a model based on x-ray diffraction data and second harmonic-generated images. Biomech Mod Mechanobiol. 2013;12:1101-1113.

5. Pandolfi A, Vasta M. Fiber distributed hyperelastic modeling of biological tissues. Mech Mater. 2012;44:151-162.

6. Gizzi A, Pandolfi A, Vasta M. A generalized statistical approach for modelling fiber-reinforced materials. J Eng Math. 2017;1-16. doi:10.1007/s10665-017-9943-5.

7. Abass A, Hayes S, White N, Sorensen T, Meek KM. Transverse depth-dependent changes in corneal collagen lamellar orientation and distribution. J Roy Soc Interface. 2015;12:20140717.

8. Sacks MS. Incorporation of experimentally-derived fiber orientation into a structural constitutive model for planar collagenous tissues. J Biomech Eng - Trans ASME. 2003;125:280-287.

9. Lanir Y. Constitutive equations for fibrous connective tissues. J Biomech. 1983;16:1-12. 
10. Organ CO, Saccomandi G. A new constitutive theory for fiber-reinforced incompressible nonlinearly elastic solids. J Mech Phys Solids. 2005;53:1985-2015.

11. Federico S, Herzog W. Towards an analytical model of soft tissues. J Biomech. 2008;41:3309-3313.

12. Gasser TC, Ogden RW, Holzapfel GA. Hyperelastic modeling of arterial layers with distributed collagen fibre orientations. J Roy Soc Interface. 2006;3:15-35.

13. Federico S, Gasser TC. Nonlinear elasticity of biological tissues with statistical fibre orientation. J Roy Soc Interface. 2010;7:955-966.

14. Vasta M, Gizzi A, Pandolfi A. On three-and two-dimensional fiber distributed models of biological tissues. Prob Eng Mech. 2014;37:170-179.

15. Gizzi A, Pandolfi A, Vasta M. Statistical characterization of the anisotropic strain energy in soft materials with distributed fibers. Mech. Mater. 2016;92:119-138.

16. Hashlamoun K, Grillo A, Federico S. Efficient evaluation of the material response of tissues reinforced by statistically oriented fibres. ZAMP. 2016;67:113.

17. Holzapfel GA, Niestrawska JA, Ogden RW, Reinisch AJ, Schriefl AJ. Modelling non-symmetric collagen fibre dispersion in arterial walls. J Roy Soc Interface. 2015;12:20150188.

18. Gizzi A, Vasta M, Pandolfi A. Modeling collagen recruitment in hyperelastic bio-material models with statistical distribution of the fiber orientation. Int J Eng Sci. 2014;78:48-60.

19. Latorre M, Montáns FJ. On the tension-compression switch of the Gasser-Ogden-Holzapfel model: Analysis and a new pre-integrated proposal. J Mech Behav Biomed Mater. 2016;57:175-189.

20. Li K, Ogden RW, Holpzafel GA. Computational method for excluding fibers under compression in modeling soft fibrous solids. Eur J Mech A/Solids. 2016;57:178-193.

21. Maceri F, Marino M, Vairo G. A unified multiscale mechanical model for soft collagenous tissues with regular fiber arrangement. J Biomech. 2010;43:355-363.

22. Marino M, Vairo G. Stress and strain localization in stretched collagenous tissues via a multiscale modelling approach. Comput Method Appl Mech Eng. 2014;17:11-30. 